\title{
Intercomparison of ion beam analysis software for the simulation of backscattering spectra from two-dimensional structures
}

\author{
M. Mayer ${ }^{\mathrm{a}}$, P. Malinskýb,c, F. Schiettekatte ${ }^{\mathrm{d}}$, Z. Zolnai ${ }^{\mathrm{e}}$
}

\author{
${ }^{\mathrm{a} M a x-P l a n c k-I n s t i t u t ~ f u ̈ r ~ P l a s m a p h y s i k, ~ B o l t z m a n n s t r . ~ 2, ~ D 85748 ~ G a r c h i n g, ~ G e r m a n y ~}$ \\ ${ }^{b}$ Nuclear Physics Institute of the Czech Academy of Sciences v.v.i., 25068 Rez, Czech \\ Republic
}

${ }^{\mathrm{c} D}$ Department of Physics, Faculty of Science, J. E. Purkinje University, Ceske mladeze 8, 400 96 Usti nad Labem, Czech Republic

${ }^{\mathrm{d}}$ Regroupement Québécois sur les Matériaux de Pointe (RQMP), Département de Physique, Université de Montréal, Montréal, QC, Canada

${ }^{\mathrm{e}}$ Centre for Energy Research, Institute of Technical Physics and Materials Science (MFA), Konkoly-Thege M. út 29-33, H-1121 Budapest, Hungary

\begin{abstract}
The codes RBS-MAST, STRUCTNRA, F95-Rough and CORTEO are simulation codes for ion beam analysis spectra from two- or three-dimensional sample structures. The codes were intercompared in a code-code comparison using an idealized grating structure and by comparison to experimental data from a silicon grating on tantalum interlayer. All codes are in excellent agreement at higher incident energies and not too large energy losses. At lower incident energies, grazing angles of incidence and/or larger energy losses plural scattering effects play an increasing role. Simulation codes with plural scattering capabilities offer higher accuracy and better agreement to experimental results in this regime.
\end{abstract}




\section{Introduction}

The quantitative application of ion beam analysis (IBA) methods such as Rutherford backscattering (RBS), elastic backscattering (EBS), elastic recoil detection analysis (ERDA), medium energy ion scattering (MEIS) and non-resonant nuclear reaction analysis (NRA) [1] requires the application of computer simulation codes for quantitative evaluation of the measured spectra [2, 3]. Available simulation codes for IBA and the implemented physics therein have been reviewed about a decade ago [2]. All codes assume laterally homogeneous samples where the distribution of elements varies only with depth, i.e. perpendicular to the surface, but is invariant in lateral directions, i.e. parallel to the surface. The sample is divided into horizontal slabs with variable thicknesses and compositions, different elements are assumed to be atomically mixed in each slab. Concentrations of elements then can be described as function of depth by concentration depth profiles. An intercomparison of seven different simulation code packages showed very good agreement between the codes for these types of samples [4, 5].

In practice, however, often laterally inhomogeneous samples are analyzed using IBA methods. Laterally inhomogeneous samples are all kinds of two- or three-dimensional structures and include all types of rough layers, surfaces and interfaces; nanoparticles, nanopillars or other nanostructures; porous materials; or compound multi-phase materials like geological samples, sinter materials, paint, or collections of dust particles. These laterally inhomogeneous samples constitute new challenges for simulation codes. Several models with different levels of generality have been developed for the simulation of IBA spectra from rough substrates or rough layers $[6,7,8,9,10,11,12,13,14,15,16,17,18,19,20]$. A model for porous materials with random distribution of small spherical pores is described in [21]. MEIS spectra from three-dimensional nanostructures at the surface of a substrate can be simulated using the program PowerMeis [22]. 
The pioneering multi-purpose code for the simulation of RBS spectra from arbitrary two- or three-dimensional sample structures was RBS-MAST [23]. Recently the program STRUCTNRA was developed [24]: It allows simulating IBA spectra from arbitrary twodimensional sample structures and uses the SIMNRA code [25] as simulation kernel. F95Rough [26] is a code for the simulation of common RBS spectra including surface roughness as 2D cuts of the measured sample. The Monte-Carlo code CORTEO [27] has been recently extended to use two- or three-dimensional sample structures as input [28]. RBS-MAST [23], STRUCTNRA [24] and F95-Rough [26] are analytical codes [29] approximating incident and exit trajectories as straight lines connected by a single scattering event. RBS-MAST combined with the DEPTH code [30] and STRUCTNRA can additionally take into account the effects of multiple small-angle scattering approximately as additional energy spread contribution [30, 31]. Lateral deflections are neglected. STRUCTNRA can additionally calculate plural large-angle scattering in the dual scattering approximation [32] using an averaged one-dimensional target structure. CORTEO [27] is a Monte-Carlo code with weight function and takes all effects of plural scattering into account. This paper is organized as follows: Participating codes and the used input parameters are shortly described in section 2. Details of the experimental setup are given in section 3. A code-code comparison for an idealized target structure is presented in section 4, results of code calculations are compared to experimental data in section 5.

\section{Simulation codes}

\subsection{RBS-MAST}

RBS-MAST is a Monte-Carlo simulation program for calculating RBS spectra taken on 2D or 3D structured samples. The code has been introduced by Z. Hajnal and co-workers for the analysis of porous structures [23] and was developed further by F. Pászti et al. and recently by E. Kótai at the Research Institute for Particle and Nuclear Physics, Wigner Research Centre 
for Physics in Budapest, Hungary. In RBS-MAST both periodic and randomly distributed structures can be treated. The individual ions are followed both inward and outward as if they would jump in the sample by steps of random length along straight trajectories.

The sample is built up from subsequent layers each constructed from elementary cells. Inside the cells objects of various shape, size and relative positions can be embedded, i.e. spheres, cylinders, bricks, ellipsoids, cones, or wedges of arbitrary atomic composition can be placed. Periodic structures can be treated by applying periodic boundary conditions for the elementary cell, while random structures are modeled by properly shifting and/or tilting the elementary cells when the ion passes through the cell walls.

The program first calculates a raw spectrum that takes into consideration only the geometric effects caused by the 3D structure of the sample. To add the classical energy spread contributions, the resulted spectrum can be convolved with a response function with given full width at half maximum (FWHM). In this work a Gaussian-type response function was used, its FWHM was calculated as a function of depth with the DEPTH code [30] using an averaged one-dimensional target structure. In the DEPTH code the energy resolution of the detector and Chu + Yang electronic energy loss straggling [33] were used. Optionally, the effect of multiple scattering was also taken into account as additional energy spread contribution [30]. In all RBS-MAST simulations presented here Rutherford cross-sections with Andersen screening [34] and SRIM-2003 stopping powers [35] were used. Note, RBS-MAST previously has been applied in the 3D structure analysis of porous materials [23], non-continuous layers [36], fish otholits [37], nanoparticles, and surfaces patterned by nanosphere lithography [38, 39, 40].

\subsection{SIMNRA/STRUCTNRA}

The STRUCTNRA program is described in detail in [24]. It allows the simulation of RBS, ERDA, NRA and MEIS spectra from arbitrary two-dimensional sample structures. The 
sample is defined by an image with quadratic pixels of adjustable size. This image can be an artificial drawing of the sample cross-section or a scanning or transmission electron microscopy image. Each color represents a material or can be empty. Strictly speaking he image is the intersection of the plane spawned by incident and exit beams with the sample and is not necessarily perpendicular to the sample surface. Periodic boundary conditions are applied in horizontal direction. The starting points of incident trajectories are distributed quasi-randomly. SIMNRA 6.98 [25, 41] was used as simulation kernel. For the presented simulations Rutherford cross-sections with Andersen screening [34] were used. SRIM-2008 stopping powers [35] and Chu + Yang electronic energy loss straggling [33] were used. STRUCTNRA assumes trajectories of incident and outgoing particles to be straight. Multiple small-angle scattering is taken into account approximately as additional energy spread [30, 31], lateral spread is neglected. Dual large-angle scattering [32] is approximated in slab geometry, with the composition of each slab as average composition of all material pixels in one row. Simulations with perpendicular orientation of the grating, see section 4 , were performed using STRUCTNRA. Simulations with parallel orientation of the grating were performed using the general roughness algorithm of SIMNRA [20, 41].

\subsection{F95-Rough}

The basic idea of the F95-Rough code is to generate a large number of particular RBS spectra for the ion beam impinging the sample surface at different, randomly chosen entrance points and scattered under identical conditions. The sum of all particular spectra simulates the real spectrum from the rough sample. The calculation is performed using $2 \mathrm{D}$ profiles provided as a perpendicular cut of the sample surface/interface. For the sake of simplicity the surface/interface profile is represented by a polyline consisting of points and line segments between consecutive points. The trajectories of the incident and emerging ions are approximated by straight lines and the slowdown process is separated from the scattering 
events. Multiple and plural scattering are not taken into account. The length of the ion trajectory, measured from first particle entrance point, is incremented by a small, constant step (can be liberally chosen) and the penetrating ion energy is changed accordingly using the appropriate stopping powers particularly valid for the investigated local ion position. For this purpose SRIM stopping powers [35] and Bohr straggling [42] are used. At each incremental step during the simulation the current ion coordinates are tested if they are positioned in the sample surface or in the underlying substrate in order to decide what physical parameters as cross sections, kinematic factors and related parameters should be used for the simulation. At each incident ion position virtual scattering into the direction to the detector is considered with the probability proportional to the appropriate Rutherford cross section with L'Écuyer screening [43]. The ingoing ion is removed from the simulation as the ion reaches the sample back side or the ion energy falls below some the predefined value. The outgoing trajectory of the scattered ion is treated in the same manner.

In such a way for each ion trajectory a full RBS spectrum is obtained, respecting ion entrance point coordinates and the local appropriate sample properties. Each ion generates the RBS spectrum by the procedure described above and is stored in a virtual multichannel analyzer. The calculation is repeated many times with randomly generated ion entrance points spread over a representative sample area to obtain sufficient statistics covering as much as possible the surface morphological artifacts distribution. At last, the final RBS spectrum is summed over all ions and is convoluted with a Gaussian function containing the assumed detector resolution with energy spread estimated with the code DEPTH [30] for the mean layer thickness. To take into account the fine features of the sample surface properly, typically several hundred ion trajectories have to be generated. The simulation of about 500 trajectories using $2 \mathrm{MeV} \mathrm{He}^{+}$ions as projectiles requires approximately $600 \mathrm{~s}$ on a standard PC computer.

\subsection{CORTEO}


Corteo [27] simulates RBS, ERD, and coincidence spectra through Monte Carlo methods, i.e. by simulating the detailed trajectory of each ion down to a certain depth, where a main scattering collision occurs, and then the trajectory of the emerging ion, within a cone about the detector. Hence, multiple/plural scattering is computed in details, including in targets that consist of 2D or 3D pixel or voxel image on top of an arbitrarily thick homogeneous layer. As for STRUCTNRA, this image can be derived from a microscopy image, each color representing a material or vacuum. Images corresponding to an implantation or an interdiffusion depth profiles, for instance, can be produced. See Ref. [28] for details. A mode called Full-RBS is also available where the main scattering approximation is avoided, but is computationally intensive. A hybrid, reasonably fast mode is also possible [44] but was not used here. Periodic boundary conditions apply along the horizontal dimensions. The beam and detector can be oriented arbitrarily with respect to the target and to each other. All simulations presented here assume Rutherford cross-sections with Andersen screening [34]. SRIM-2008 stopping powers [35] and Chu + Yang electronic energy loss straggling [33] are used.

\section{Experimental}

All measurements were performed at the tandem accelerator of IPP Garching with $1000 \mathrm{keV}$ and $1500 \mathrm{keV}$ incident ${ }^{4} \mathrm{He}^{+}$ions. The scattering angle was $165^{\circ}$ in Cornell geometry. The detector was a PIPS detector having a resolution of $20 \mathrm{keV}$ FWHM. The solid angle was determined experimentally using four certified reference materials from IRMM and was $(1.108 \pm 0.038)$ msr. The detector aperture is a slit with a height of $1 \mathrm{~mm}$ and a width of $5 \mathrm{~mm}$ with rounded corners having a radius of $0.5 \mathrm{~mm}$. The nominal aperture - sample distance is $64.1 \mathrm{~mm}$.

The sample was a silicon grating on top of a tantalum interlayer on top of bulk silicon. A cross-section of the grating structure using focused ion beam (FIB) cross-sectioning is shown in Fig. 1. The lateral distance of the trenches was about $500 \mathrm{~nm}$ with a trench width of about 
$250 \mathrm{~nm}$. Based on the scanning electron microscopy (SEM) images the thickness of the top silicon grating was $272.3 \mathrm{~nm}$ for the trenches and $447.1 \mathrm{~nm}$ for the heights, the thickness of the tantalum interlayer was $680 \mathrm{~nm}$. The accuracy of these numbers is about $\pm 10 \mathrm{~nm}$. Backscattering spectra were recorded with the grating structure parallel to the plane formed by incident and exit beams, see Fig. 2 (left), and perpendicular to that plane, see Fig. 2 (right). The orientation of the sample with respect to the plane formed by incident and exit beams was accurate within about $\pm 1^{\circ}$. The sample was rotated between $0^{\circ}$ and $70^{\circ}$ along the thick dashed line in Fig. 2 (right).

The energy calibration was performed by measuring the high-energy edges of bulk Au, Rh, Co, Al, and C targets at $1000 \mathrm{keV}$ and $1500 \mathrm{keV}$ energy. The energy calibration was slightly non-linear. The offset and the linear and quadratic terms were found to be $64.4 \mathrm{keV}, 2.675$ $\mathrm{keV} / \mathrm{ch}$, and $-2.944 \times 10^{-4} \mathrm{keV} / \mathrm{ch}^{2}$, respectively.

\section{Code-code comparison}

For the code-code comparison an idealized sample was used and is shown in Fig. 3. It has periodic boundary conditions in horizontal direction. The Si and Ta layers have natural mass densities of $2.322 \mathrm{~g} / \mathrm{cm}^{3}$ and $16.60 \mathrm{~g} / \mathrm{cm}^{3}$, respectively. The Si substrate was neglected in order to allow faster calculations. RBS-MAST, SIMNRA/STRUCTNRA and CORTEO used Andersen screening to the Rutherford cross-section [34], F95-Rough used L’Écuyer screening [43]. At backscattering angles above about $150^{\circ}$ both screening functions are almost identical. All codes used SRIM-2003 or SRIM-2008 stopping powers [35]. These are identical for single elements. Chu + Yang electronic energy loss straggling [33] was used by SIMNRA/STRUCTNRA and CORTEO, and in RBS-MAST in combination with the DEPTH code, while F95-Rough used Bohr straggling [42]. Particles*sr was set to $10^{10}$, a linear energy calibration with offset $0 \mathrm{keV}$ and $1 \mathrm{keV} / \mathrm{channel}$ and a detector resolution of $15 \mathrm{keV}$ were used. 
SIMNRA/STRUCTNRA, RBS-MAST and F95-Rough used infinitesimally small detectors.

CORTEO used a detector size of $1 \times 4.8 \mathrm{~mm}^{2}$, located at $64.1 \mathrm{~mm}$ from the beam spot for most simulations. Some simulations were performed with a detector size of $1 \mathrm{x} 1 \mathrm{~mm}^{2}$, located at $150 \mathrm{~mm}$ from the beam spot (small detector).

\subsection{Single scattering}

Simulated spectra using single scattering are shown in Fig. 4 for parallel and perpendicular orientation of the grating for 1000, 1500 and $2000 \mathrm{keV}$. Single scattering means that multiple and plural scattering (if available in the code) were not considered. CORTEO assumed nuclear collisions to result only in energy transfer to recoils, but not in angular deflections of the projectile, i.e. nuclear collisions resulted in nuclear energy loss and nuclear energy spread, but not in angular spread: This is identical to single scattering in the other codes. As described in section 2.2 the general roughness algorithm of SIMNRA was used for the parallel grating orientation, while STRUCTNRA was used for the perpendicular orientation of the grating. The simulated spectra show mainly the backscattering signal from Ta. The Si backscattering signal can be recognized for example in the energy range from 600 to $850 \mathrm{keV}$ in the $1500 \mathrm{keV}$ simulation (note the small step from the Si surface at about $850 \mathrm{keV}$, see Fig. 4 middle row), but it overlaps with the backscattering signal from Ta and is therefore hardly visible. The high-energy edge of Ta is shifted towards lower energies due to the overlying Si layer, the shape of this edge reflects the lateral and depth distribution of Si.

For parallel orientation of the grating (Fig. 4 left row) the high-energy edge of Ta shows two steps due to the two different thicknesses of the overlying Si layer. In single scattering approximation there are no lateral deflections, so that incident and exiting particles always penetrate the same Si layer thickness. The resulting spectrum is therefore the linear superposition of two spectra with two different thicknesses of Si. Correlations (for example 
incidence through the thin Si layer and exit through the thick Si layer) do not occur within this approximation.

For perpendicular orientation of the grating (Fig. 4 right row) the Ta edge shows three steps. This is due to particle trajectories with incidence and exit through the thin Si layer; with incidence and exit through the thick Si layer; and trajectories with incidence through the thick Si layer and exit through the thin layer or the side wall of the trench; and trajectories with incidence through the thin Si layer and exit through the thick layer or through the edge of the height. The latter two types of trajectories result in the intermediate step.

All four codes calculate spectra with only minor differences. The high-energy Ta edge is calculated almost identical by all four codes, i.e. for smaller energy losses and low-Z targets all codes provide comparable results. SIMNRA/STRUCTNTRA and RBS-MAST are generally in excellent agreement also at larger energy losses. F95-Rough is in very good agreement with SIMNRA/STRUCTNTRA and RBS-MAST for smaller energy losses, but shows some small deviations at higher energy losses. CORTEO is in excellent agreement with SIMNRA/STRUCTNTRA and RBS-MAST when using a small point-like detector, see the dashed yellow line in Fig. 4 for $1500 \mathrm{keV}$ parallel orientation (the line is hardly visible because it overlaps with SIMNRA/STRUCTNTRA and RBS-MAST), but requires long computing times. The use of a realistic detector (also used in Section 5) results in a discrepancy at the high- and low-energy Ta edges especially for parallel orientation (see Fig. 4 for the 1500 and $2000 \mathrm{keV}$ parallel simulations). This results from the possibility that particles have to cross sidewalls when considering a finite size detector instead of crossing only two thicknesses, and a fraction of the particles are able to reach the detector after traveling through a range of effective thicknesses.

\subsection{Multiple and plural scattering}


Simulated spectra taking plural scattering effects into account are shown in Fig. 5. CORTEO takes all multiple/plural scattering effects (i.e. energy spread by multiple or plural collisions as well as the effect of traversing different materials due to lateral deflections) into account. RBS-MAST (in combination with DEPTH) and SIMNRA/STRUCTNRA use the analytical theory of multiple small-angle scattering from [30, 31] as additional energy spread contribution, but effects of lateral deflections are neglected. SIMNRA/STRUCTNRA additionally approximate plural large-angle scattering in the dual scattering approximation [32]. For two-dimensional structures dual scattering is further approximated in slab geometry, where the composition of each slab is the average composition of all material pixels in one row.

Taking only multiple small-angle scattering into account (dashed black and red lines in Fig. 5) shows very good agreement between SIMNRA/STRUCTNRA and RBS-MAST. Only at $1000 \mathrm{keV}$ incident energy the low-energy edge of Ta shows some differences, which is probably due to a slightly different implementation of electronic energy-loss straggling (and potentially also multiple scattering) at large energy losses.

Plural scattering effects, as calculated by CORTEO, are small at $2000 \mathrm{keV}$. Simulated spectra including plural scattering effects are not significantly different from spectra in single scattering approximation at this energy, see section 4.1. Plural scattering plays a visible role only at low energies below about $200 \mathrm{keV}$. At 1000 and $1500 \mathrm{keV}$ incident energy plural scattering effects play a larger role and modify the spectra by a steeper increase of the Ta signal towards lower energies, add a tail at energies below the Ta low-energy edge, see Fig. 5 yellow lines, and result in an additional smearing of the low-energy Ta edge (see the $1500 \mathrm{keV}$ case in Fig. 5). SIMNRA/STRUCTNRA shows astonishingly good agreement with the CORTEO results (yellow lines in Fig. 5) when approximating plural scattering by multiple plus dual scattering (solid black lines in Fig. 5), especially at 1500 and $2000 \mathrm{keV}$. The steeper increase of the Ta signal towards lower energies as well as the tail at energies below the Ta 
low-energy edge are well described: The dual-scattering approximation with averaged target therefore can extend the accuracy of simulations using the single-scattering approximation towards lower energies.

The additional smearing of the low-energy Ta edge in the CORTEO spectra (especially in the $1500 \mathrm{keV}$ cases in Fig. 5) is not reproduced by RBS-MAST and SIMNRA/STRUCTNRA. This smearing may be partly due to the finite detector size used by CORTEO. However, it remains visible also with a smaller detector (dashed yellow lines in Fig. 5) and is therefore probably caused by lateral deflections due to multiple small-angle scattering: Particles close to the side walls of the trenches can escape from the material by small-angle deflections. This effect of lateral spread cannot be calculated accurately by RBS-MAST and SIMNRA/STRUCTNRA but requires full Monte-Carlo simulations.

\section{Comparison to experimental data}

Experimental spectra were simulated using the target structure shown in Fig. 6. This target structure is derived as mean value based on several FIB cross-sections (see Fig. 1 as example) and on SEM images, but also on fits to the RBS spectra by varying the two different thicknesses of the Si layer. Experimental spectra from the grating structure are shown in Fig. 7 for $1000 \mathrm{keV}$ incident energy and in Fig. 8 for $1500 \mathrm{keV}$ incident energy. The measurements were performed in Cornell geometry at different rotation angles for parallel and perpendicular orientation of the grating, see Fig. 2 for the geometry. The angle of incidence is measured towards the surface normal.

The general roughness algorithm of SIMNRA [41] was used to simulate the spectra with parallel grating orientation [20], while STRUCTNRA was used for the perpendicular orientation. CORTEO used a detector size of $1 \times 4.8 \mathrm{~mm}^{2}$, located at a distance of $64.1 \mathrm{~mm}$ from the beam spot: This is identical to the experimental conditions. 
RBS-MAST was combined with the DEPTH code in order to account for detector resolution, stopping-induced straggling, and the effect of small angle multiple scattering as additional energy spread contribution. The sample tilt angle was also taken into account in the DEPTH calculations.

At $1500 \mathrm{keV}$ CORTEO, SIMNRA/STRUCTNRA, RBS-MAST and F95-Rough reproduce the experimental data generally very well. SIMNRA/STRUCTNRA without dual scattering (dashed black line in Figs. 7 and 8) RBS-MAST (red line) and F95-rough (blue line) are close together but slightly underestimate the experimental data at channels below about 200 . SIMNRA/STRUCTNRA gives an improved fit to the experimental data if dual scattering is included (solid black line in Figs. 7 and 8), especially for the lower energetic part of the spectra. CORTEO also fits the data very well, but shows some statistical fluctuations. At $1000 \mathrm{keV}$ SIMNRA/STRUCTNRA and F95-Rough fit the experimental data very well at incident angles of $0^{\circ}$ and $45^{\circ}$. At $60^{\circ}$ the edge in channels around 150 starts to be too steep in the simulations. This effect is even more pronounced at $70^{\circ}$, where the simulated edge in channels around 70 is too steep and does not well reproduce the experimental data. RBSMAST gives very good agreement with the experimental data at all tilt angles. The shape of the spectra calculated by CORTEO fits all experimental data very well.

\section{Summary and conclusions}

The codes RBS-MAST, SIMNRA/STRUCTNRA, F95-Rough and CORTEO are simulation codes for RBS-spectra from two- or three-dimensional sample structures. RBS-MAST, SIMNRA/STRUCTNRA and F95-Rough are analytical single-scattering codes with varying abilities to take multiple- and plural-scattering effects approximately into account. CORTEO is a Monte-Carlo code including all types of plural scattering effects. The codes were intercompared in a code-code comparison using an idealized structure of a grating and by comparison to experimental data from a silicon grating on tantalum interlayer. 
All four codes are in very good agreement if the spectra can be approximated by the singlescattering approximation. This is the case at higher energies, low-Z materials and not too large energy losses. Some minor differences between the codes are observed at larger energy losses, probably due to different implementations of energy-loss calculations and the evaluation of electronic energy-loss straggling and energy straggling by multiple scattering (if implemented in the code). At lower energies the single scattering approximation fails gradually and plural scattering effects play an increasing role. The plural scattering contribution can be described astonishingly well by the analytical theory of multiple scattering from [30, 31] plus the dual scattering approximation from [32] with averaged target. Only the low-energy edge of rough layers is not well reproduced by these approximations. Measurements at grazing angles of incidence are not perfectly reproduced by F95-Rough and STRUCTNRA, while RBS-MAST combined with the DEPTH code showed very good agreement. Monte-Carlo simulations using CORTEO provide a very good fit to the experimental data, but are subject to statistical fluctuations.

Computing times for the simulation of spectra from two-dimensional structures can be considerably higher than from samples in slab geometry and vary considerably between the different codes. Realistic detectors (as can be used in CORTEO) can result in geometryinduced smearing of edges. The consideration of this effect can increase the accuracy of simulations, but also limits the possibilities for calculational speed-ups by using large virtual detectors in Monte-Carlo simulations. Single scattering codes currently implement only infinitesimally small detectors. Depending on the real detector size in the experimental setup one or the other approximation is more suitable.

All four codes are generally suitable for the simulation of backscattering spectra from rough surfaces. Main physics differences between the codes are the implementation of multiple and plural scattering effects. At sufficiently high incident beam energies, small energy losses in the sample and/or low-Z targets multiple and plural scattering effects play only a minor role. 
In this case all investigated codes yield very similar results. At lower incident energies, larger energy losses in the sample or gracing angles of incidence codes with plural scattering capabilities (i.e. STRUCTNRA and CORTEO and with some trade-offs RBS-MAST) offer higher accuracy and better agreement to experimental data.

The simulation of heterogeneous materials, such as porous samples, randomly dispersed nanoparticles, paint [45], or geological samples, is generally possible with all four codes, where each code uses a different model of the sample. The above discussion of multiple and plural scattering effects applies also for these kind of samples.

Scanning electron microscopy (SEM) in combination with focused ion beam cross-sectioning (FIB) and transmission electron microscopy (TEM) allows deriving the three-dimensional sample structure. However, these methods are destructive and usually provide only information about a limited area of the sample while ion beam analysis methods provide statistical information about a relatively large sample area. In this respect these methods are complementary.

\section{Acknowledgements}

SEM and FIB investigations by G. Matern and the technical assistance with accelerator measurements by M. Fußeder and J. Dorner are gratefully acknowledged. The silicon grating was generously provided by K. Krieger. P. Malinsky is grateful for the financial support provided by Grant agency project P108/12/G108. Z. Zolnai appreciates partial support from the Hungarian scientific research grant OTKA K 112114. F. Schiettekatte thanks Calcul Québec for computing resources, and financial support NSERC and FRQ-NT. 


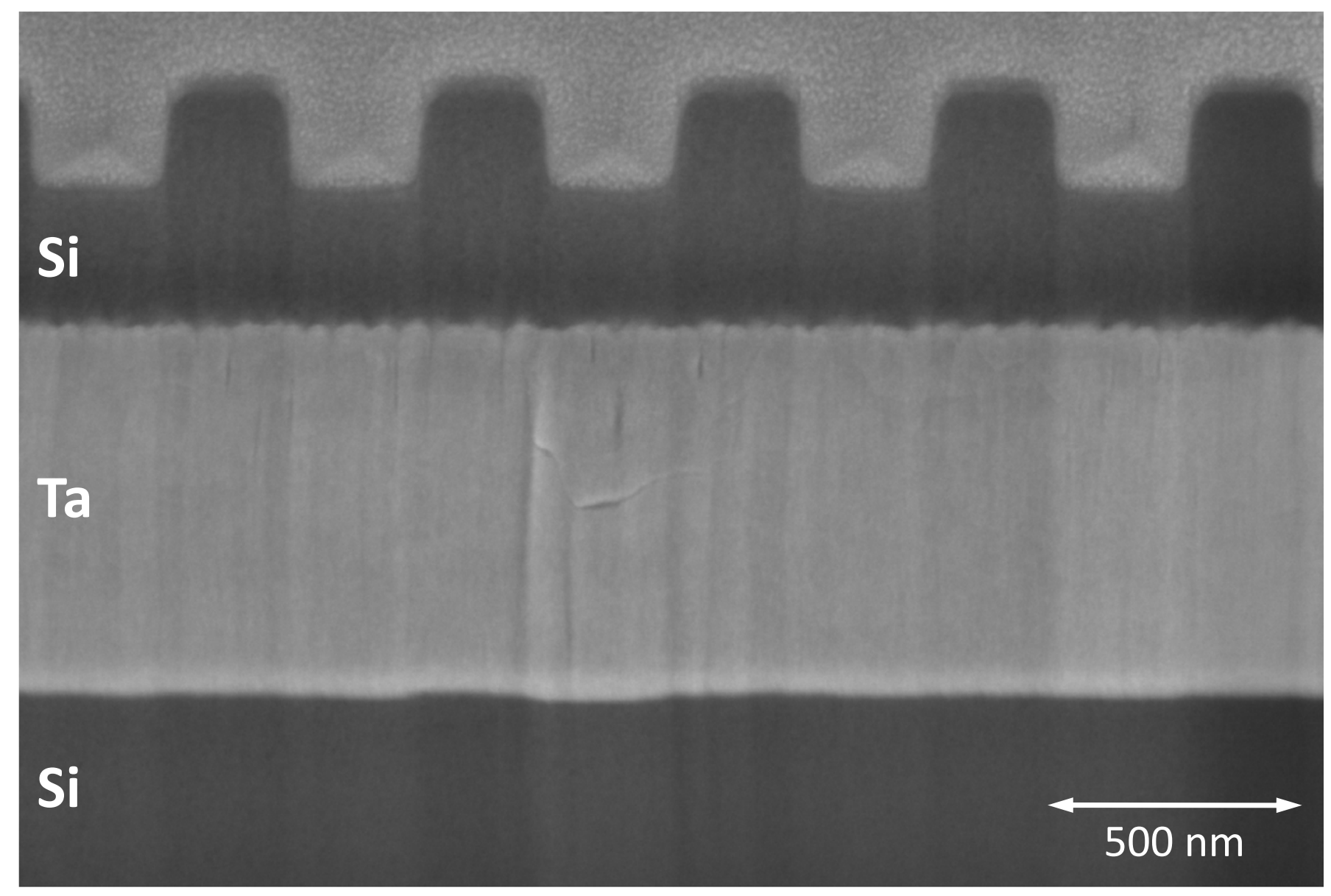

Fig. 1: Focused ion beam cross-section through the silicon grating sample consisting of a structured silicon layer on top of a tantalum interlayer on top of silicon substrate. 

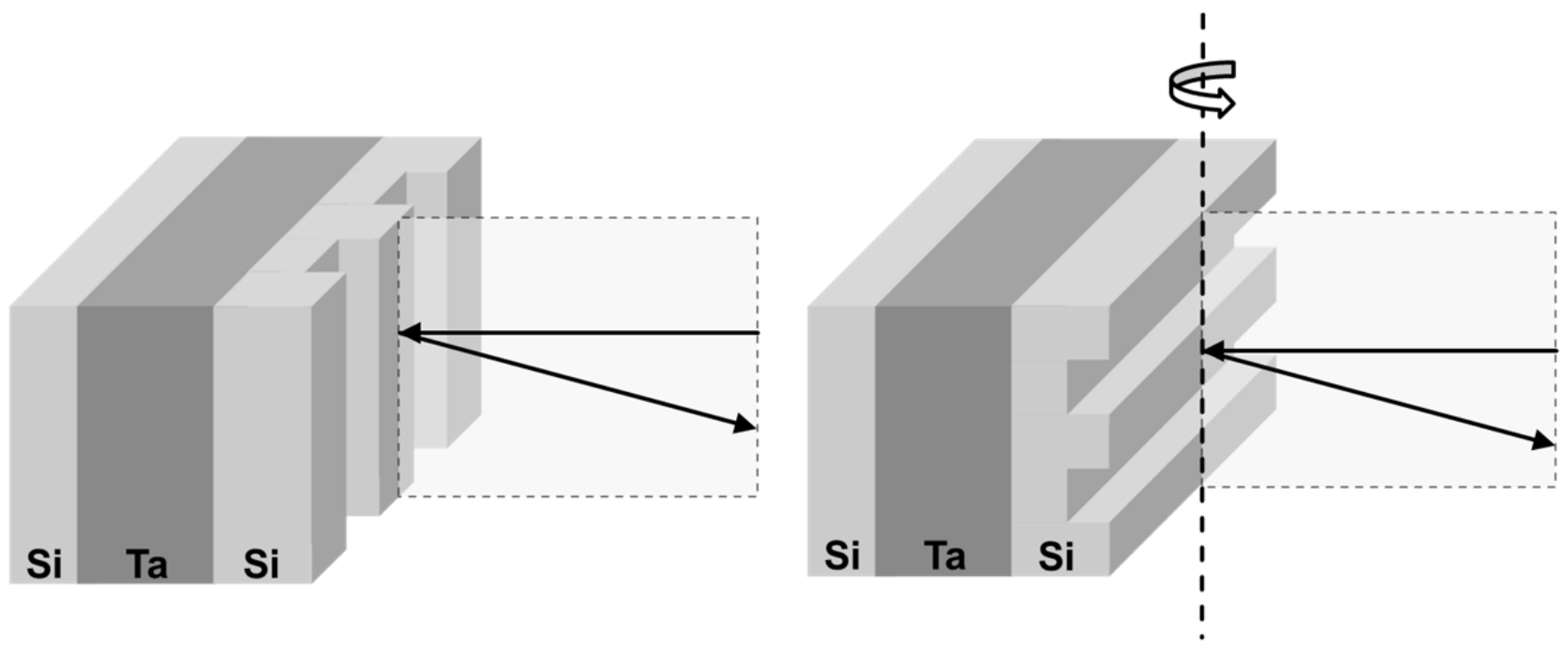

Fig. 2: Geometry of the measurements with the grating parallel to the plane formed by incident and exit beams (left) and perpendicular to this plane (right). The thick dashed line is the rotation axis of the sample for measurements at oblique incidence. 


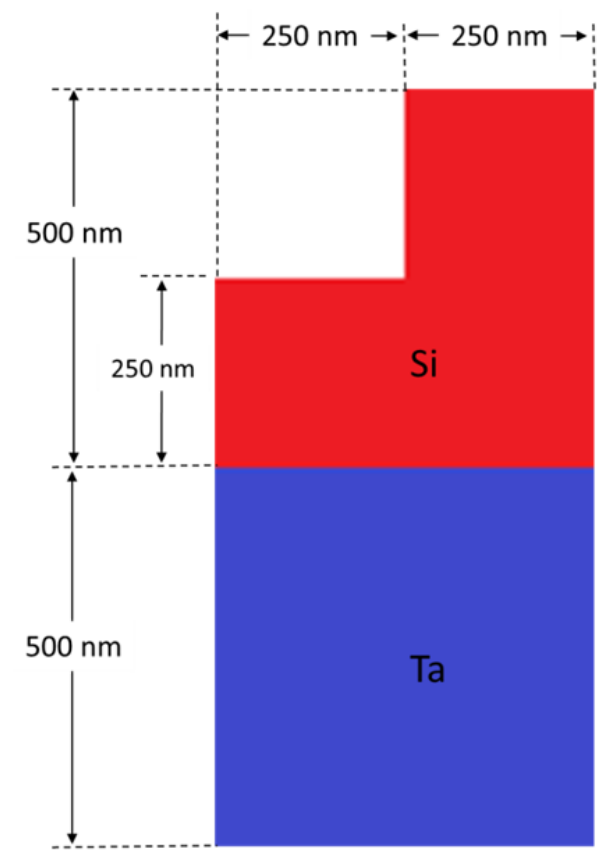

Fig. 3: Idealized sample structure used for the code-code comparison. The sample has periodic boundary conditions in horizontal direction, Si and Ta have natural mass densities. 

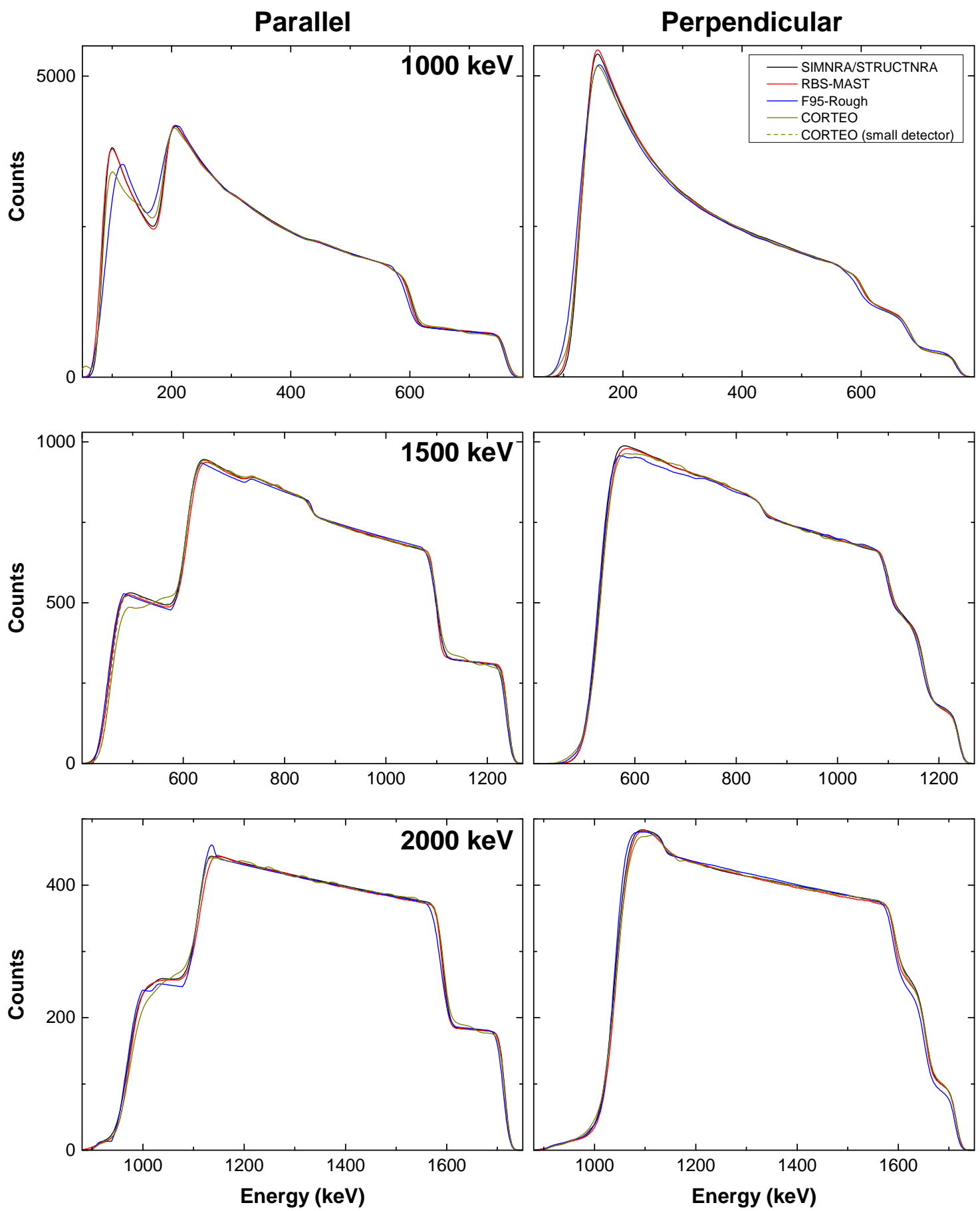

Fig. 4: Simulated spectra for 1000, 1500 and $2000 \mathrm{keV}{ }^{4} \mathrm{He}$ backscattered at normal incidence from the Si/Ta target shown in Fig. 3 in single-scattering approximation. Left column: Parallel orientation of the grating, see Fig. 2 left; Right column: Perpendicular orientation, see Fig. 2 right. Top row: $1000 \mathrm{keV}$ incident energy; Middle row: $1500 \mathrm{keV}$; Bottom row: $2000 \mathrm{keV}$. Solid black lines: SIMNRA/STRUCTNRA; Solid red lines: RBS-MAST; Solid blue lines: F95-Rough; Solid yellow lines: CORTEO using single scattering and large detector; Dashed yellow line: CORTEO using single scattering and small detector (only for 1500 keVparallel orientation). 

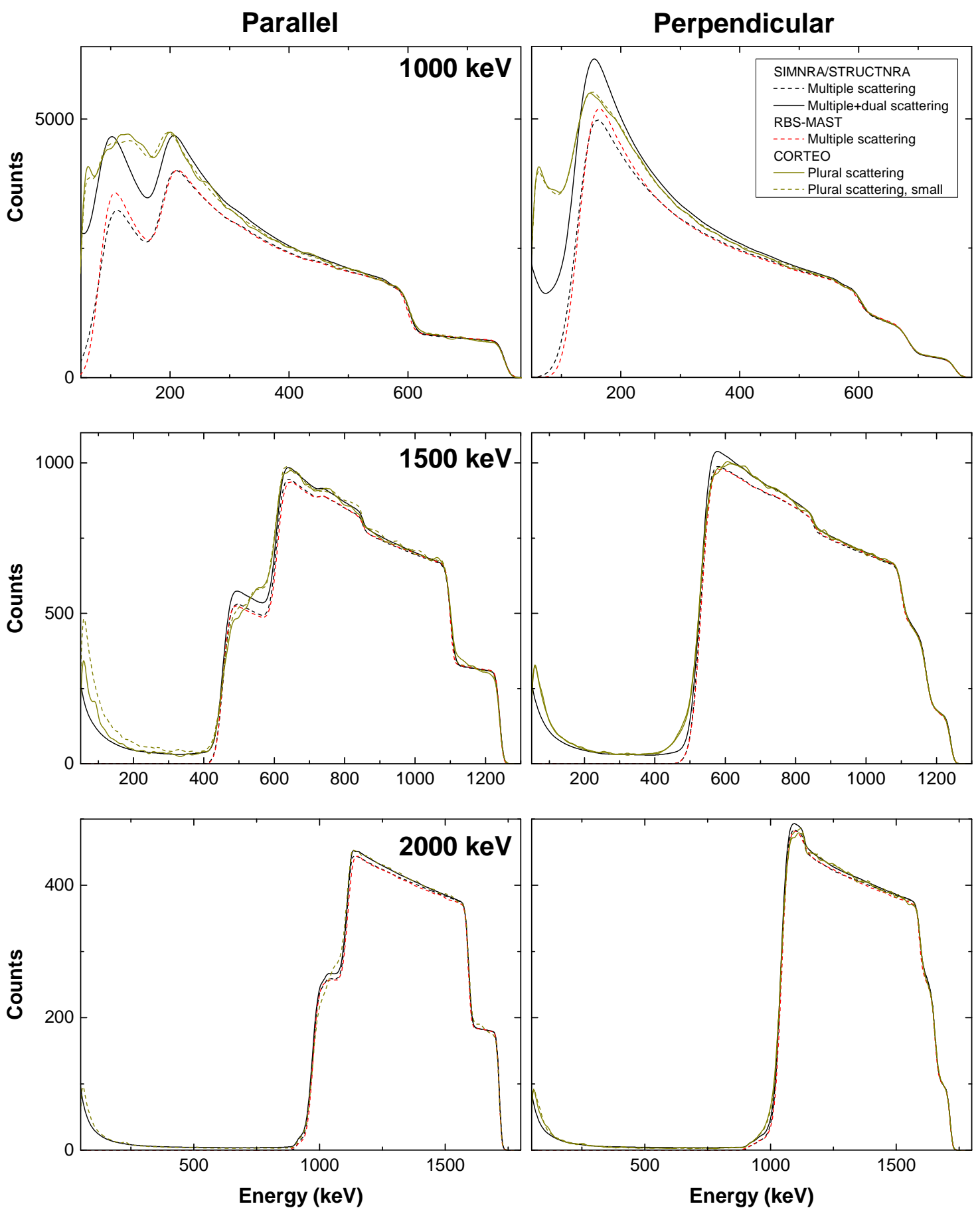

Fig. 5: Simulated spectra for 1000, 1500 and $2000 \mathrm{keV}{ }^{4} \mathrm{He}$ backscattered at normal incidence from the Si/Ta target shown in Fig. 3 including multiple and plural scattering. Left column: Parallel orientation of the grating, see Fig. 2 left; Right column: Perpendicular orientation, see Fig. 2 right. Top row: $1000 \mathrm{keV}$ incident energy; Middle row: $1500 \mathrm{keV}$; Bottom row: $2000 \mathrm{keV}$. Dashed black lines: SIMNRA/STRUCTNRA using multiple small-angle scattering induced energy spread; Solid black lines: SIMNRA/STRUCTNRA using multiple small-angle scattering induced energy spread plus dual scattering; Dashed red lines: RBS-MAST using multiple small-angle scattering induced energy spread; Solid yellow lines: CORTEO using all plural scattering contributions; Dashed yellow lines: CORTEO using all plural scattering contributions with a smaller detector. 


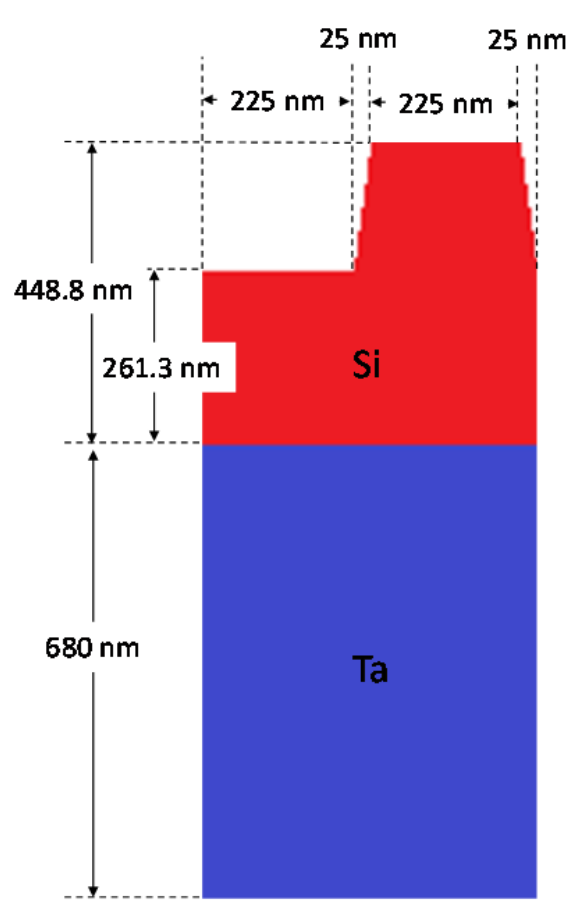

Fig. 6: Sample used for the comparison to experimental data. The sample has periodic boundary conditions in horizontal direction, Si and Ta have natural mass densities. 


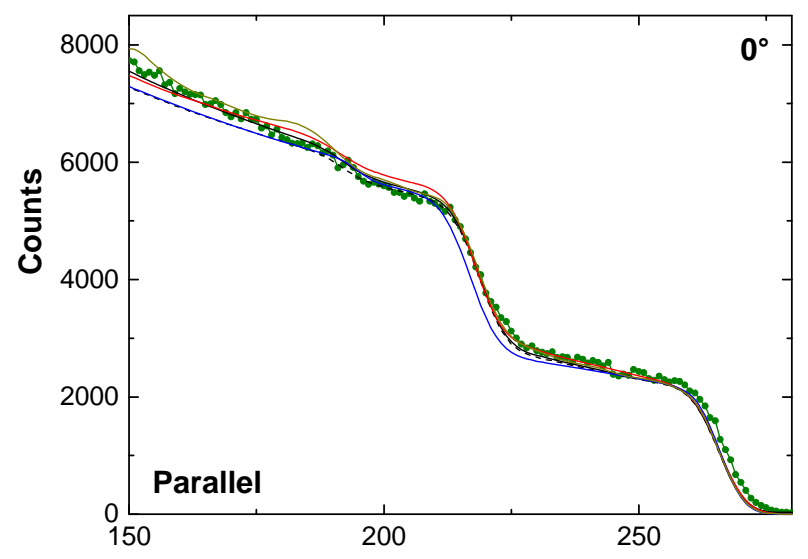

\section{$1000 \mathrm{keV}$}
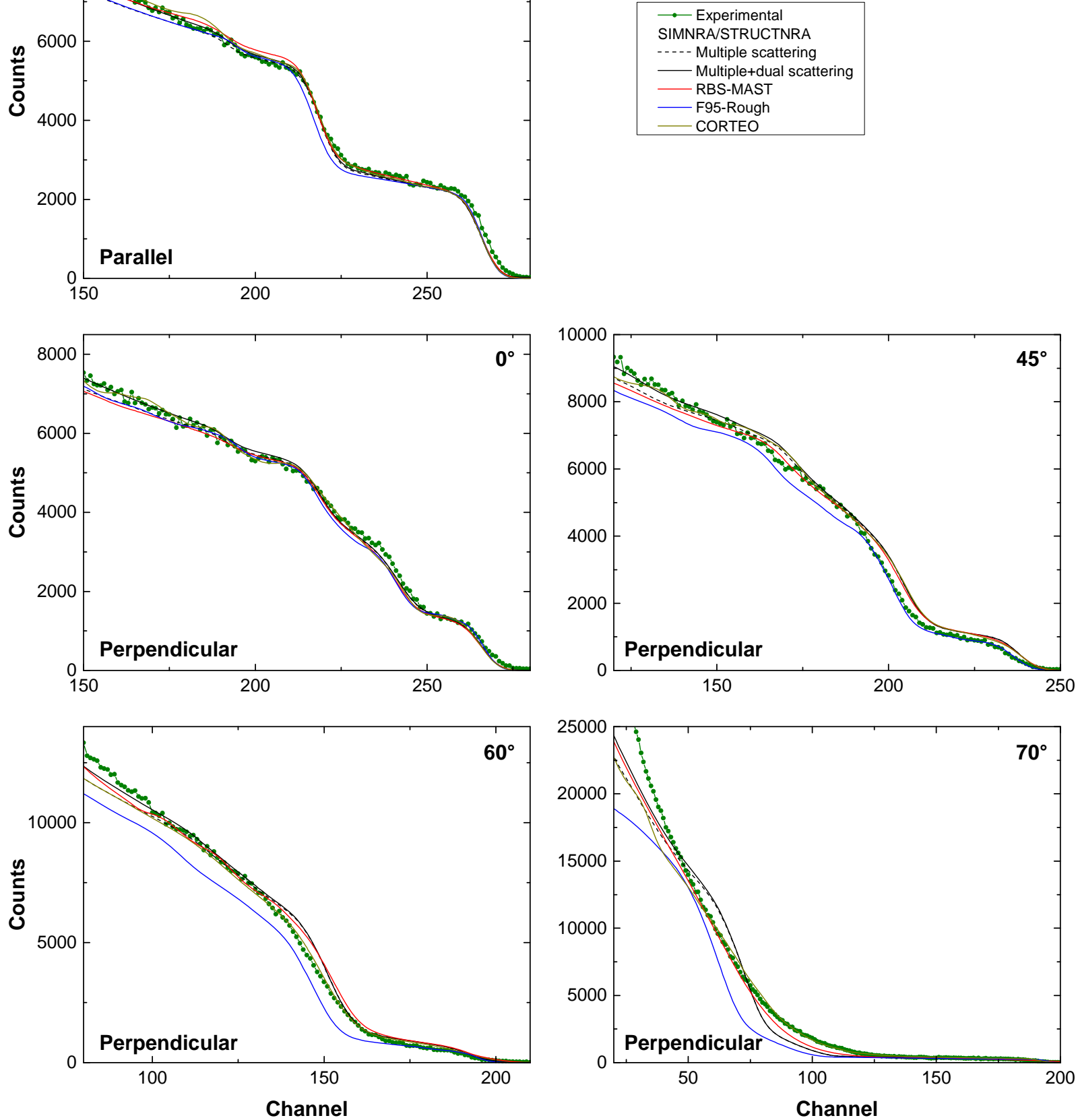

Fig. 7: Experimental and simulated backscattering spectra from the silicon grating shown in Fig. 1 using $1000 \mathrm{keV}^{4} \mathrm{He}$ ions at incident angles from $0^{\circ}$ to $70^{\circ}$. Top left: Parallel orientation of the grating at $0^{\circ}$, see Fig. 2 left. All other: Perpendicular orientation of the grating. The angle of incidence is indicated. Green symbols: Experimental data; Dashed black lines: SIMNRA/STRUCTNRA with multiple small-angle scattering; Solid black lines: SIMNRA/STRUCTNRA with multiple small-angle scattering plus dual scattering; Solid red line: RBS-MAST; Solid blue line: F95-Rough; Solid yellow lines: CORTEO. 

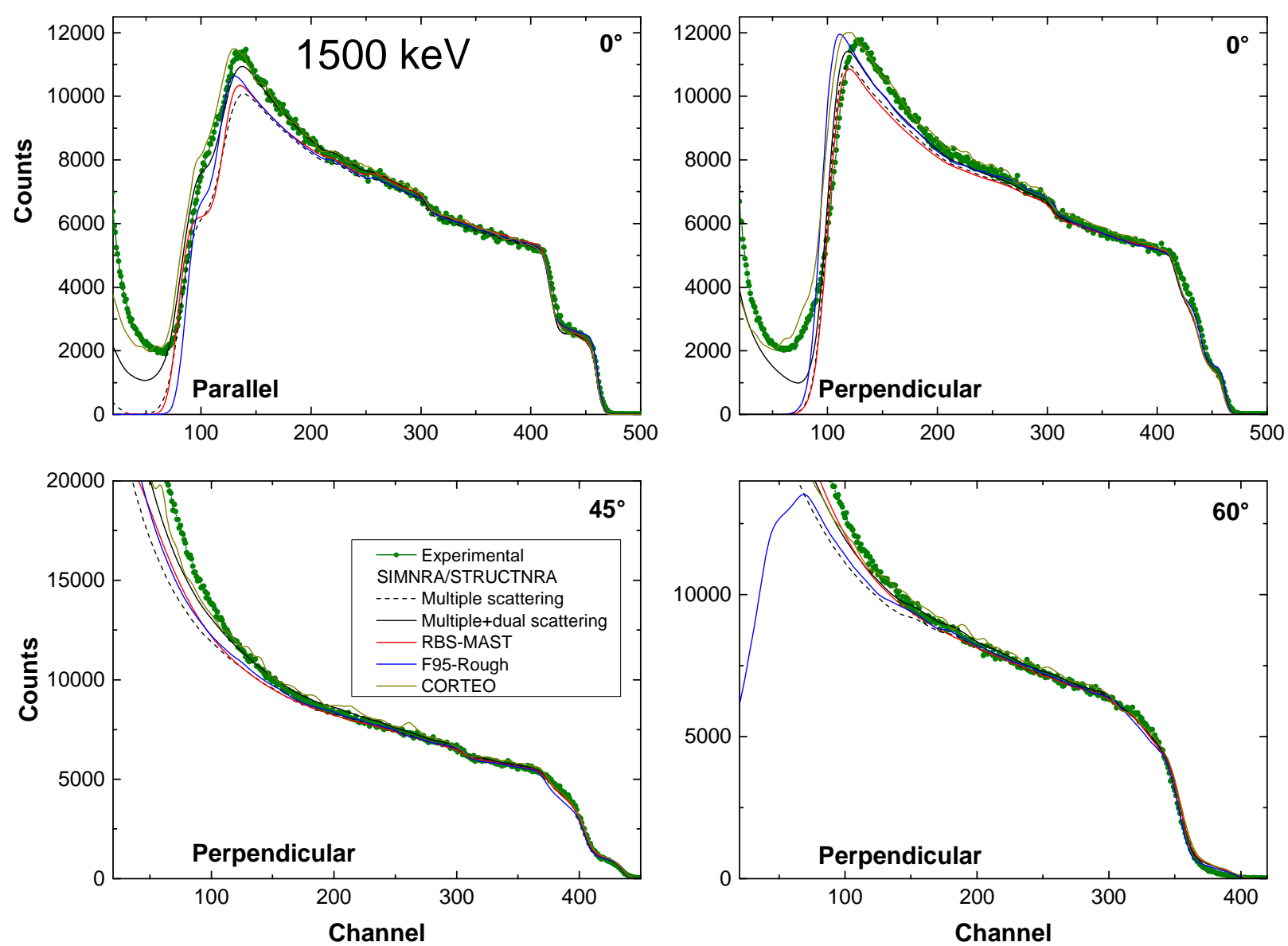

Fig. 8: Experimental and simulated backscattering spectra from the silicon grating shown in Fig. 1 using $1500 \mathrm{keV}^{4} \mathrm{He}$ ions at incident angles from $0^{\circ}$ to $60^{\circ}$. Top left: Parallel orientation of the grating at $0^{\circ}$, see Fig. 2 left. All other: Perpendicular orientation of the grating. The angle of incidence is indicated. Green symbols: Experimental data; Dashed black lines: SIMNRA/STRUCTNRA with multiple small-angle scattering; Solid black lines: SIMNRA/STRUCTNRA with multiple small-angle scattering plus dual scattering; Solid red line: RBS-MAST; Solid blue line: F95-Rough; Solid yellow lines: CORTEO. 
1 J.R. Tesmer, M. Nastasi, Handbook of Modern Ion Beam Materials Analysis, Materials Research Society, Pittsburgh, Pennsylvania, 1995

2 E. Rauhala, N.P. Barradas, S. Fazinic, M. Mayer, E. Szilágyi, M. Thompson, Nucl. Instrum. Methods B 244 (2006) 436

3 N.P. Barradas, M. Mayer, M.A. Reis, F.Schiettekatte, IBA Software, Chapter 15 of the book Ion Beam Analsis: Fundamentals and Applications, M. Nastasi, J.W. Mayer, Y. Wang (eds.), CRC Press, Taylor and Francis Group, 2015, ISBN 9781439846384

4 N.P. Barradas, K. Arstila, G. Battistig, M. Bianconi, N. Dytlewski, C. Jeynes, E. Kótai, G. Lulli, M. Mayer, E. Rauhala, E. Szilágyi, M. Thompson, Nucl. Instrum. Methods B 262 (2007) 281

5 N.P. Barradas, K. Arstila, G. Battistig, M. Bianconi, N. Dytlewski, C. Jeynes, E. Kotai, G. Lulli, M. Mayer, E. Rauhala, E. Szilagyi, M. Thompson, Nucl. Instrum. Methods B 266 (2008) 1338

6 R.D. Edge, U. Bill, Nucl. Instrum. Methods 168 (1980) 157

7 A.R. Knudson, Nucl. Instrum. Methods 168 (1980) 163

8 J.R. Bird, P. Duerden, D.D. Cohen, G.B. Smith, P. Hillery, Nucl. Instrum. Methods 218 (1983) 53

9 C.P. Hobbs, J.W. McMillan, D.W. Palmer, Nucl. Instrum. Methods B 30 (1988) 342

10 M. Wüest, P. Bochsler, Nucl. Instrum. Methods B 71 (1992) 314

11 V.S. Shorin, A.N. Sosnin, Nucl. Instrum. Methods B 72 (1992) 452

12 H. Metzner, M. Gossla, Th. Hahn, Nucl. Instrum. Methods B 124 (1997) 567

13 I.M. Yesil, W. Assmann, H. Huber, K.E.G. Löbner, Nucl. Instrum. Methods B 136-138 (1998) 623

14 A. Kitamura, T. Tamai, A. Taniike, Y. Furuyama, T. Maeda, N. Ogiwara, M. Saidoh, Nucl.

Instrum. Methods B 134 (1998) 98

15 H. Metzner, Th. Hahn, M. Gossla, J. Conrad, J.-H. Bremer, Nucl. Instrum. Methods B 134 (1998) 249

16 T. Sajavaara, K. Arstila, A. Laakso, J. Keinonen, Nucl. Instrum. Methods B 161-163 (2000) 235

17 E. Edelmann, K. Arstila, J. Keinonen, Nucl. Instrum. Methods B 228 (2005) 364

18 M. Mayer, Nucl. Instrum. Methods B 194 (2002) 177

19 S.L. Molodtsov, A.F. Gurbich, C. Jeynes, J. Phys. D 41 (2008) 205303

20 H. Langhuth, M. Mayer, S. Lindig, Nucl. Instrum. Methods B 269 (2011) 1811

21 M. Mayer, U. von Toussaint, J. Dewalque, O. Dubreuil, C. Henrist, R. Cloots, F. Mathis, Nucl. Instrum. Methods B 273 (2012) 83

22 M.A. Sortica, P.L. Grande, G. Machado, L. Miotti, J. Appl. Phys. 106 (2009) 114320

23 Z. Hajnal, E. Szilágyi, F. Pászti, G. Battistig, Nucl. Instrum. Methods B 118 (1996) 617

24 M. Mayer, Nucl. Instrum. Methods B 371 (2016) 90

25 M. Mayer, SIMNRA User’s Guide, Tech. Rep. IPP 9/113, Max-Planck-Institut für Plasmaphysik, Garching, 1997

26 P. Malinský, V. Hnatowicz, A. Macková, Nucl. Instrum. Methods B 371 (2016) 101

27 F. Schiettekatte, Nucl. Instrum. Methods B 266 (2008) 1880

28 F. Schiettekatte, M. Chicoine, Nucl. Instrum. Methods B 371 (2016) 106 
29 M. Mayer, W. Eckstein, H. Langhuth, F. Schiettekatte, U. von Toussaint, Nucl. Instrum. Methods B 269 (2011) 3006

30 E. Szilágyi, F. Pászti, G. Amsel, Nucl. Instrum. Methods B 100 (1995) 103

31 G. Amsel, G. Battistig, A. L’Hoir, Nucl. Instrum. Methods B 201 (2003) 325

32 W. Eckstein, M. Mayer, Nucl. Instrum. Methods B 153 (1999) 337

33 Q. Yang, D.J. O’Connor, Z. Wang, Nucl. Instrum. Methods B 61 (1991) 149

34 H.H. Andersen, F. Besenbacher, P. Loftager, W. Möller, Phys. Rev. A21(6) (1980) 1891

35 J.F. Ziegler, Nucl. Instrum. Methods B 219-220 (2004) 1027

36 Á.Tunyogi, F. Tanczikó, Cs. Bogdán, Z. E. Horváth, and E. Szilágyi, Nucl. Instrum. Methods B 268 (2010) 1972

37 R. Huszánk, A. Simon, E. Szilágyi, K. Keresztessy, and I. Kovács, Nucl. Instrum. Methods B 267 (2009) 2132

38 Z. Zolnai, A. Deák, N. Nagy, A.L. Tóth, E. Kótai, and G. Battistig, Nucl. Instrum. Methods B 268 (2010) 79

39 Z. Zolnai, N. Nagy, A. Deák, G. Battistig, and E. Kótai, Phys. Rev. B 83 (2011) 233302

40 Z. Zolnai, Appl. Surf. Sci. 281 (2013) 17

41 M. Mayer, Nucl. Instrum. Methods B 332 (2014) 176

42 N. Bohr, Philos. Mag. 30 (1915) 581

43 J. L’Ecuyer, J.A. Davies, N. Matsunami, Nucl. Instrum. Methods 160 (1979) 337

44 F. Schiettekatte, Nucl. Instrum. Methods B 332 (2014) 404

45 M. Mayer, T.F. Silva, Computer Simulation of Backscattering Spectra from Paint, presented at ECAART 12, submitted to Nucl. Instrum. Methods B 